\title{
Santilli Synthesis of the Neutron According to Hadronic Mechanics
}

\author{
Chandrakant S. Burande \\ Vilasrao Deshmukh College Engineering and Technology, Mouda, Dist. Nagpur, India
}

\section{Email address:}

csburande@gmail.com

\section{To cite this article:}

Chandrakant S. Burande. Santilli Synthesis of the Neutron According to Hadronic Mechanics. American Journal of Modern Physics. Special Issue: Issue II: Foundations of Hadronic Mechanics. Vol. 5, No. 2-1, 2016, pp. 37-45. doi: 10.11648/j.ajmp.2016050201.13

Received: July 17, 2015; Accepted: July 18, 2015; Published: May 18, 2016

\begin{abstract}
In 1920 H. Rutherford conjectured that neutron is a compressed hydrogen atom in the core of the stars. W. Pauli noted that such synthesis of neutron violates the conservation of the angular momentum. Therefore, E. Fermi proposed emission of massless particle, called "neutrino". However, R.M. Santilli more recently noted that, even though the angular angular momentum would be conserved, the neutrino hypothesis does not allow non-relativistic quantum mechanics to be valid because the rest energy of the neutron is bigger than the sum of the rest energies of the proton and electron, under these conditions Schrodinger equation becomes inconsistent. Similarly, Santilli showed that relativistic quantum mechanics is also inapplicable (rather than violated) because, even though exactly valid for the electron at large distance from the proton in the hydrogen atom, the celebrated Dirac's equation is clearly inapplicable for the representation of electron when immersed inside the proton. In this paper, we study Santilli's decades of mathematical, theoretical and experimental research, first for the construction of the covering hadronic mechanics, and then the resulting numerically exact and time invariant representation at the non-relativistic and relativistic levels of "all" chararcteristics of the neutron in its synthesis from a proton and an electron. In particular, we show that, within said covering context, the representation of proton as an extended particle implies the existence of an orbital angular momentum of the electron within the hyperdense proton which is totally non-existence for quantum mechanics, under which the total angular momentum is conserved without any need for the conjectural neutrino. We finally study Santilli's suggestive hypothesis of the "etherino" as a longitudinal impulse (rather than particle) from the ether as a universal substratum that delivers missing energy for the synthesis of the neutron.
\end{abstract}

Keywords: Neutron, Binding Energy, Isoelectron, Hulthen Potential, Lie-Santilli Isoalgebras

\section{Introduction}

In 1920, Rutherford [1] submitted the hypothesis that hydrogen atoms in the core of stars are compressed into new neutral particles having the size of the proton that he called neutrons (Figure 1), according to the synthesis

$$
p^{+}+e^{-} \rightarrow n
$$

The existence of the neutron was confirmed in 1932 by Chadwick [2]. However, Pauli [3] noted that the spin $1 / 2$ of the neutron cannot be represented via a quantum state of proton and electron, each having spin $1 / 2$. Fermi [4] adopted Pauli's objection and, he then developed the theory of weak interactions according to which the synthesis of the neutron is characterized by either the emission of a neutral and massless particle, named neutrino $(v)$ or absorption of antineutrino $(\bar{v})$. The particle reactions as per proposed theory of weak interaction are given by

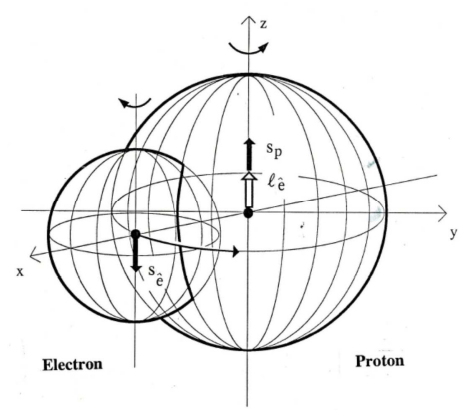

Figure 1. A schematic view of the the deep overlapping of the wavepackets of electrons into hyperdense medium of proton with singlet coupling. 


$$
\begin{gathered}
p^{+}+e^{-} \rightarrow n+v, \quad \text { or } \\
p^{+}+e^{-}+\bar{v} \rightarrow n .
\end{gathered}
$$

However, Santilli [5-7] has dismissed the Fermi's version of synthesis of neutron on following grounds:

1. the sum of the rest energies of the proton and of the electron,

$$
\begin{aligned}
m_{p}+m_{e} & =938.272 \mathrm{MeV}+0.511 \mathrm{MeV} \\
& =938.783 \mathrm{MeV}
\end{aligned}
$$

is smaller than the rest energy of the neutron,

$$
m_{n}=939.565 \mathrm{MeV}
$$

with positive energy (binding energy) difference of 0.78 $\mathrm{MeV}$,

2. Schrödinger equation does not admit positive binding energy for quantum bound states when electron totally immersed within the hyper-dense medium inside the proton structure,

3. classical theory of antimatter requires that the anti-neutrino has a negative energy, although, eq.(3) is needed positive energy to supply the missing energy, $0.78 \mathrm{MeV}$,

4. neither, antineutrino can deliver the $0.78 \mathrm{MeV}$ needed for the neutron synthesis because the cross section of former with electron or proton is null, and

5. the proton and the electron are the only experimentally discovered stable massive particles. Hence, emission of neutrino in neutron formation does not have any relevance. Moreover, it cannot be directly detected.

\section{Hadronic Energy}

The only bound state of a proton and an electron predicted by quantum mechanics is the hydrogen atom, with smallest orbit (Bohr's orbit) of the order of $10^{-8} \mathrm{~cm}$. Santilli's hadronic mechanics has identified the existence of an additional bound state when the electron orbits within the proton structure at distances of the order of $10^{-13} \mathrm{~cm}$ or less. The difference between these two bound states is depicted in "Figure 2".

Remarkably, Santilli has proved that the hadronic state is one and only one, the neutron, and its first excited state is the Hydrogen atom which is formed when the electron leaves the proton structure, thus recovering all conventional quantum states. In this sense, the energy levels of the hydrogen atom are the excited states of the neutron.

The mutual overlapping of the charge distribution or wavepackets of electron and proton leads to new interactions of contact type. However, it is not possible via conventional quantum mechanics to represent these new interactions for various reasons, such as:

1. quantum mechanics can only represent particles as dimensionless point masses; quantum mechanics has a local-differential structure ruling out any consistent treatment of the nonlocal integral interactions;

2. quantum mechanics can only represent interactions derivable from a local potential, while contact interactions of the type required to be considered herein can be represented with anything except a potential or a Hamiltonian.

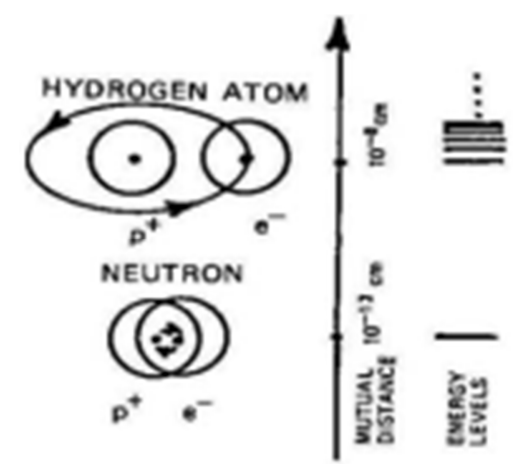

Figure 2. A schematic comparison of Bohr's orbit and hadronic structure identified by Santilli.

In this event, Santilli's isomechanics is ideally suited for a quantitative study of the neutron synthesis because, in addition to all interactions characterizing the hydrogen atom, it allows the new interactions caused by deep mutual penetration of the constituents. This method has been used by Santilli in numerous applications. Santilli [5-8] obtained an isoequation for the neutron by isotopically lifting of Schrödinger equation introducing additional potential term of Coulomb nature that reads as,

$$
\begin{aligned}
& {\left[\frac{1}{m} \hat{p} \times \hat{T} \times \hat{p} \times \hat{T}-\frac{z e^{2}}{r}+\frac{e^{2}}{r} \hat{T}\right] \times \hat{\psi}(r)} \\
& =E \times \hat{\psi}(r)
\end{aligned}
$$

with isounit,

$$
\hat{I}=U \times I \times U^{\dagger}=1 / \hat{T}>0 .
$$

The suitable isounit to represent the two particle penetration (now termed as an isoelectron), is defined as, with isounit,

$$
\begin{aligned}
\hat{I} & =\operatorname{Diag}\left(n_{1}^{2}(1), n_{2}^{2}(1), n_{3}^{2}(1), n_{4}^{2}(1)\right) \\
& \times \operatorname{Diag}\left(n_{1}^{2}(2), n_{2}^{2}(2), n_{3}^{2}(2), n_{4}^{2}(2)\right) \\
& \times \exp \left((\psi / \hat{\psi}) \times \int \hat{\psi}(r)_{1 \downarrow}^{?} \times \widehat{\psi}(r)_{2 \uparrow} d r\right)
\end{aligned}
$$

where the two diagonal matrices represent the shapes (assumed to be spheroids) and the densities of the particles considered, while the last term represents the non-Hamiltonian interactions. For spherical point-like charge particle, such as electrons, the diagonal matrices get reduced to 1 . Next, the evaluation of the volume integral into a constant,

$$
N=\int \widehat{\psi}(r)_{1 \downarrow}^{?} \times \widehat{\psi}(r)_{2 \uparrow} d r
$$


and the expansion of the isoexponent up to the second term, yields,

$$
\begin{aligned}
& \hat{I} \approx e^{N \times \psi / \hat{\psi}} \approx 1+N \times \psi / \widehat{\psi} \\
& \hat{T} \approx e^{-N \times \psi / \hat{\psi}} \approx 1-N \times \psi / \hat{\psi} \\
& |\hat{I}| \gg 1, \quad|\hat{T}| \ll 1, \lim _{r \rightarrow 1 f m} \hat{I}=1 .
\end{aligned}
$$

In above equations $\psi$ and $\widehat{\psi}$ behave respectively as

$$
\begin{gathered}
\psi \approx P \times e^{-b r} \\
\widehat{\psi} \approx Q \times\left(1-e^{-b r}\right) / r
\end{gathered}
$$

where $P$ and $Q$ are constants and $b$ is inverse of hadronic horizon, $r_{h}$. Using eqs.(13) and (14), the isotopic element depicted in eq.(11) reads as

$$
\hat{T} \approx 1-N \times \psi / \widehat{\psi}=1-r \times V_{0} \frac{e^{-b r}}{\left(1-e^{-b r}\right)},
$$

where $V_{0}=N P / Q$. Now, by introducing Hulthen potential,

$$
V_{\text {Hulthen }}=V_{0} \frac{e^{-b r}}{1-e^{-b r}}
$$

where $V_{0}$ is the Hulthen's constant, the isotopic element can be written as

$$
\hat{T} \approx 1-N \times \psi / \widehat{\psi}=1-r \times V_{\text {Hulthen }} .
$$

Further, at small distances, the Hulthen potential behaves like Coulomb potential,

$$
V_{\text {Hulthen }} \approx \frac{V_{0}}{b \times r} .
$$

which is very strong as the quantity $b$ in the denominator is of the order of $10^{-13} \mathrm{~cm}$, thus resulting the multiplicative factor of the order of $10^{13}$. As a result, inside the hadronic horizon, the Coulomb potential is absorbed by the Hulthen potential, thus we can write

$$
\begin{aligned}
& \frac{e^{2}}{r} \times \hat{T}-\frac{z e^{2}}{r} \\
& \approx \frac{e^{2}}{r} \times\left(1-r \times V_{\text {Hulthen }}\right)-\frac{z e^{2}}{r} \\
& =-V \times \frac{e^{-b r}}{\left(1-e^{-b r}\right)}
\end{aligned}
$$

where $z=1$ and $V=e^{2} V_{0}$.

Using eqs.(6) and (19), Santilli obtained the nonrelativistic radial isoequation of the hadronic two-body structure model that reads as

$$
\begin{aligned}
& {\left[\frac{1}{r^{2}}\left(\frac{d}{d r} r^{2} \frac{d}{d r}\right)\right] \times \hat{\psi}(r)} \\
& +\left[\frac{m}{\rho^{2} \hbar^{2}}\left(E_{h b}+V \frac{e^{-b r}}{1-e^{-b r}}\right)\right] \times \hat{\psi}(r)=0
\end{aligned}
$$

where $E_{h b}$ is hadronic binding energy. Assuming the change of variable, $x=1-e^{-h r}$, eq.(20) can be written as

$$
\begin{aligned}
& {\left[x(1-x) \frac{d^{2}}{d x^{2}}\right] S(x)} \\
& -\left[\left(2|A|^{1 / 2}+1\right) \frac{d}{d x}+\beta^{2}\right] S(x)=0
\end{aligned}
$$

where

$$
\begin{aligned}
& k_{2}=\frac{m V_{0}}{\hbar^{2} \rho^{2} b^{2}}, \\
& A=\frac{m}{\hbar^{2} \rho^{2} b^{2}} E_{b}<0,
\end{aligned}
$$

The solution of eq.(21) is then given by

$$
\begin{aligned}
& G_{n}(x) \\
& =\sum_{k=1}^{k=n}\left(\begin{array}{l}
n-1 \\
k-1
\end{array}\right)\left(\begin{array}{c}
n+k+2|A|^{1 / 2}-1 \\
k
\end{array}\right) x^{k}
\end{aligned}
$$

with isonormalized isoeigenfunction

$$
\begin{aligned}
\widehat{\psi}(r) & =\left[\frac{\Gamma\left(2|A|^{1 / 2}+3\right)}{\Gamma(3) \Gamma\left(2|A|^{1 / 2}\right)}\right]^{1 / 2} \\
& \times \frac{1-e^{-b r}}{r} e^{-|A|^{1 / 2} b r}
\end{aligned}
$$

the expression for hadronic binding energy is then obtained as

$$
\left|E_{h b}\right|=E^{\text {Bind }}=\frac{V_{0}}{4 k_{2}}\left[\frac{k_{2}}{n}-n\right]^{2} .
$$

The boundary conditions demand that $k_{2}>n$. This indicates the finite value of eigenvalues for Hulthen potential. This is in concurrence with the hadronic bound state. Further, for an isoparticle to be bounded inside the hadronic horizon $b^{-1}$, its wavelength, $\lambda$ must be proportional to the horizon itself, and we shall write

$$
\lambda=\frac{1}{2 \pi k_{1} b}
$$

where $k_{1}$ is a positive quantity that must be constant for a stationary state. Next the hadronic kinetic energy $E_{h k}$ is given by 


$$
E_{h k}=\frac{\hat{p}^{2}}{2 m} \approx \frac{\hbar^{2} \rho^{2} b^{2}}{2 m} .
$$

Now, from eqs.(22) and (27), we have

$$
V_{0}=2 k_{2} E_{h k} \text {. }
$$

Hence, the hadronic total energy of the hadronic bound state is given by

$$
\begin{aligned}
E_{h t} & =2 E_{h r}+2 E_{h k}-E_{h b} \\
& \approx 2 k_{1}\left[1-\left(k_{2}-1\right)^{2}\right] \hbar b c_{0} .
\end{aligned}
$$

where $c_{0}$ is the speed of light in vacuum, and note that the last approximation holds for hadronic bound states where the rest energy is insignificant with respect to the kinetic energy. Thus, at this point we obtained the expression for the total energy of the two-body hadronic bound state which is depend on two unknown quantities, $k_{1}$ and $k_{2}$. To achieve a numerical solution, we now introduce second expression, the meanlife, $\tau$ of the unstable hadron

$$
\tau^{-1}=\lambda^{2}|\widehat{\psi}(0)|^{2} \frac{\alpha^{2} E_{h k}}{\pi \hbar},
$$

where $\alpha$ is the fine structure constant. By using the above expressions, we can write

$$
\begin{gathered}
\hat{\psi}(0)\left[\frac{1}{2} \frac{\left(k_{2}-1\right) \Gamma\left[\frac{1}{2}\left(k_{2}-1\right)+2\right]}{3 ! \Gamma\left[\frac{1}{2}\left(k_{2}-1\right)+2\right]}\right]^{1 / 2} \\
\times b=\frac{\left(k_{2}-1\right)^{3 / 2}}{(48)^{1 / 2}} b
\end{gathered}
$$

The meanlife of the hadronic bound state then becomes

$$
\tau^{-1}=\frac{4 \pi}{48(137)^{2}} \frac{\left(k_{2}-1\right)^{3}}{k_{1}} b c_{0}
$$

Thus, we obtained a system of two equations in terms of two unknown quantities $k_{1}$ and $k_{2}$, total rest energy, $E_{\text {tot }}$, the meanlife, $\tau$ and the charge radius, $R_{c}$ of the two-body hadronic bound state, that it is reproduced identically below:

$$
\begin{aligned}
& k_{1}\left[1-\left(k_{2}-1\right)^{2}\right]=\frac{E_{h t}}{2 \hbar b c_{0}} \\
& \frac{\left(k_{2}-1\right)^{3}}{k_{1}}=\frac{48(137)^{2}}{4 \pi b c_{0}} \tau^{-1} .
\end{aligned}
$$

On substituting $b=10^{-13}, \tau^{-1}=10^{-3}$ and $E_{t h}=939$ in eqs.(33) and (34), we extract

$$
k_{1}=2.6, \quad k_{2}=1+0.81 \times 10^{-8} \approx 1 .
$$

For admissible state, $n=1$, we further have

$$
\frac{k_{2}}{n}-n \approx 0,
$$

$$
E_{h b}=-\frac{V_{0}}{4 k_{2}} \times\left(\frac{k_{2}}{n}-n\right) \approx 0
$$

Thus, this proves that the in nonrelativistic approximation the hadronic binding energy is insignificant. Further, the numerical value of the hadronic kinetic energy is obtained as

$$
E_{h k} \approx k_{1} \hbar b c_{0}=6.63 \times 10^{-23} \mathrm{MeV} \approx 0
$$

which is also insignificant. The reason for being very small hadronic binding energy and ignorable in first approximation is due to the fact that contact resistive forces have no potential energy. The main physical origin of hadronic structure is the contact, zero-range, interaction due to the complete immersion of one wavepacket within the other.

Finally, Santilli arrives at the following result namely the total hadronic energy of the neutron is primarily characterized by the rest energy of the proton and the isonormalized rest energy of the isoelectron,

$$
\begin{aligned}
E_{n} & \approx E_{p}+E_{h r, \hat{e}}=E_{p}+\frac{m_{e} c_{0}^{2}}{\rho^{2}} \\
& =938.272+1.293=939.565 \mathrm{MeV}
\end{aligned}
$$

where $\rho^{2}=0.3952$ is a geometrization of the departure of the interior of hadrons from our space-time. Since the proton is not mutated in this first approximation as per our assumption, have

$$
\begin{gathered}
b_{1}=b_{2}=b_{3}=1, \\
\rho^{2}=n_{4}^{2}=b_{4}^{-2}=\frac{0.511}{1.293}=0.3952 \\
\rho=n_{4}=b_{4}^{-1}=0.6286 .
\end{gathered}
$$

Notice that the above value for the characterization of the density of the neutron coincides with the experimental value of the density of the fireball of the Bose-Einstein correlation.

\section{The Neutron Spin}

The conceptual interpretation of the observed spin $1 / 2$ of the neutron, for the first, was successfully explained by Santilli as follows. Considering the initiation of Rutherford's process of compression of the isoelectron within the proton in singlet coupling, it is evident that, as soon as the penetration begins, the isoelectron is trapped inside the hyperdense medium inside the proton, thus resulting in a constrained orbiting motion of the isoelectron that must superpose on the proton spin (FIGURE 2). Santilli stresses that the proton is not mutated because it is 2000 times heavier than the electron, and that the coupling must be in singlet for stability. This implies 
that, for the case of the neutron structure, the spin of the electron is also not mutated. However, the angular momentum of electron is mutated inside the hadronic sphere. The needed mutation of the quantum into the hadronic angular momentum is trivially given by the nonunitary transforms

$$
U \times U^{\dagger}=\hat{I}=\frac{1}{2}, \quad \hat{T}=2,
$$

The mutation is supported by the isotopic invariance of the Hilbert space. Nonunitary lifting of angular momentum, in this case, reads

$$
\begin{aligned}
& \left\langle l, m\left|\times L_{3} \times\right| l, m\right\rangle \times I \\
& \rightarrow U \times\left[\left\langle l, m\left|\times L_{3} \times\right| l, m\right\rangle\right] \times U^{\dagger} \\
& =\left\langle\hat{l}, \hat{m}\left|2 \times \hat{L}_{3} \times 2\right| \hat{l}, \hat{m}\right\rangle \times \frac{1}{2},
\end{aligned}
$$

In order to represent the spin of neutron Santilli (1990) used irregular isorepresentations of Lie-Santilli isoalgebras [9-11], namely, isorepresentations characterized by nonunitaryisounitary transforms for the generators different than those for the product. This difference is rather natural for the structure of the neutron, since the basic nonunitary transform for the rest energy has already been selected for calculation of binding energy. This irregular isopresentation of $\hat{S} O(3)$ based on the the isodifferential calculus and isolinear momentum is given below [9-11]:

$$
\begin{gathered}
{\left[\hat{r}_{i}, \hat{r}_{j}\right]=\left[\hat{p}_{i}, \hat{p}_{j}\right]=0,} \\
{\left[\hat{r}_{i} \hat{p}_{j}\right]=\hat{\delta}_{i j}=\hat{I} \times \delta_{i j}=\rho \delta_{i j}} \\
\hat{L}^{2} \hat{\times} \hat{Y}_{\hat{l} \hat{m}}(\hat{\theta}, \hat{\phi})=\rho^{2} \times \hat{l}(\hat{l}+1) \hat{Y}_{\hat{l} \hat{m}}(\hat{\theta}, \hat{\phi}), \\
\hat{L}_{3} \hat{\times} \hat{Y}_{\hat{l} \hat{m}}(\hat{\theta}, \hat{\phi})=\rho \times \hat{m} \times \hat{Y} \hat{l} \hat{m}(\hat{\theta}, \hat{\phi}), \\
\hat{l}=1,2,3, \ldots . . \quad \hat{m}=\hat{l}, \hat{l}-1, \ldots . .,-l .
\end{gathered}
$$

Notice that the isotopic lifting of the integer value of the angular momentum, $l=1,2,3, \ldots$ into the value $\rho \times \hat{l}$, where, again, $\hat{l}=1,2,3 \ldots$, the value $\hat{l}=0$ being excluded by boundary conditions, $\rho$ being a variable depending on the local conditions. For the study of the neutron spin on the line of hadronic mechanics, Santilli selected the following two-dimensional irregular isorepresentation of $\widehat{S U}(2)$ :

$$
\hat{I}=\left(\begin{array}{cc}
g_{11} & 0 \\
0 & g_{22}
\end{array}\right), \hat{T}=\left(\begin{array}{cc}
g_{11}^{-1} & 0 \\
0 & g_{22}^{-1}
\end{array}\right)
$$

$$
\begin{aligned}
& \hat{J}_{1}=\frac{1}{2}\left(\begin{array}{cc}
0 & g_{11}^{-1 / 2} \\
g_{22}^{-1 / 2} & 0
\end{array}\right), \\
& \hat{J}_{2}=\frac{1}{2}\left(\begin{array}{cc}
0 & -i g_{11}^{-1 / 2} \\
i g_{22}^{-1 / 2} & 0
\end{array}\right) \\
& {\left[\hat{J}_{1} \hat{J}_{2}\right]=i \hat{J}_{3} } \\
&=\frac{1}{2} \times \frac{\Delta^{1 / 2}}{2}\left(\begin{array}{cc}
g_{11}^{-1} & 0 \\
0 & g_{22}^{-1}
\end{array}\right) \\
&\left.\hat{J}_{3} \times|\hat{j}, \hat{s}\rangle=\hat{J}_{3} \times \hat{T}\left|\hat{j}, \hat{s}= \pm \frac{\Delta}{2}\right| \hat{j}, \hat{s}\right\rangle
\end{aligned}
$$

In this case, Santilli [9-11] has selected the two-dimensional irregular isorepresentation of $\widehat{S U}(2)$ and then computed the total angular momentum of the neutron model, $n=\left(p^{+}, \hat{e}^{-}\right)_{h m}$ as,

$$
J_{n}=J_{p}+\hat{L}_{\hat{e}}^{o r b}+\widehat{J}_{\hat{e}}^{i n t}=\frac{1}{2}+\rho-\frac{\Delta}{2}
$$

resulting in the values anticipated above, namely:

$$
\rho=\frac{1}{2}, \quad \Delta=1
$$

It shows that the spin of the isoelectron is not mutated and the angular momentum is mutated in such a way that the isoelectron is merely carried out by the proton spin.

\section{The Neutron Magnetic Moment}

In view of the hadronic orbiting motion of isoelectron, the magnetic moment of the neutron was generated by Santilli by considering the following three contributions,

$$
\mu_{n}=\mu_{p}^{i n t}-\mu_{e}^{o r b}+\mu_{\hat{e}}^{i n t}
$$

The observed values of magnetic moment of neutron and proton are respectively,

$$
\mu_{n}=-1.9 \times \frac{e}{2 m_{p} c_{0}}, \mu_{p}=2.7 \times \frac{e}{2 m_{p} c_{0}} .
$$

Now, on rearranging magnetic moment of neutron as

$$
\begin{aligned}
\mu_{n} & =-1.9 \times \frac{e}{2 m_{p} c_{0}} \\
& =2.7 \times \frac{e}{2 m_{p} c_{0}}-4.6 \times \frac{e}{2 m_{p} c_{0}},
\end{aligned}
$$

and comparing with eq.(56), we obtain following identity:

$$
-\mu_{\hat{e}}^{\text {orbital }}+\mu_{\hat{e}}^{\text {intrinsic }}=-4.6 \times \frac{e}{2 m_{p} c_{0}} .
$$


This is equivalent to

$$
\begin{aligned}
-\mu_{\hat{e}}^{\text {orbital }}+\mu_{\hat{e}}^{\text {intrinsic }} & =\frac{-2.5 \times 10^{-3} e}{2 m_{e} c_{0}} \\
& =-2.5 \times 10^{-3} \mu_{e} .
\end{aligned}
$$

From eq.(60), Santilli derived the desired value of, $\mu_{\hat{e}}^{\text {orbital }}$, that is

$$
\mu_{\hat{e}}^{\text {orbital }}=\left(1+2.5 \times 10^{-3}\right) \times \mu_{e}
$$

The small value of the total magnetic moment of the isoelectron is fully compatible with the null value of its total angular momentum.

\section{Santilli Aetherino Hypothesis}

Santilli replaces the neutrino as a physical particle in our space-time with a longitudinal impulse originated by the ether as a universal substratum that he calls "etherino" [12]. In this view, all physical quantities missing in the neutron synthesis, such as energy and spin, are delivered by said impulse.

A particular motivation for the etherino hypothesis is due to the evident difficulties in accepting that neutrino now believed to have mass could traverse entire planets and stars without appreciable scattering. By contrast, this difficulty is resolved by the propagation of a longitudinal impulse in the universal substratum because it would underlie matter.

Additionally, the replacement of the neutrino with the etherino appears to preserve the experimental evidence in the field because what is today detected and interpreted as a "neutrino scattering" could in reality be due to the scattering of the longitudinal impulse with targets.

Hence, the etherino hypothesis appears to resolve some of the insufficiencies of the neutrino conjecture, may eventually resulting to be fully compatible with available experimental data, and is already stimulating rather intriguing research on superluminal communications, that are the only possible for interstellar contact [12] due to evident insufficiencies of electromagnetic waves for galactic distances.

\section{Don Borghi Experiment on the Synthesis of Neutrons}

The first experiment on the synthesis of neutrons from protons and electrons was conducted by Carlo Borghi, C. Giori and A. Dall'Olio in the 1960 at the CEN Laboratories in Recife, Brazil [13], [14]. Hydrogen gas at fraction of 1 bar pressure was obtained from the electrolytical separation of water and was placed in the interior of a cylindrical metal chamber (called klystron) and kept mostly ionized by an electric arc with about $500 \mathrm{~V}$ and $10 \mathrm{~mA}$. Additionally, the gas was traversed by microwaves with 10 s frequency. Suitable materials which are vulnerable to nuclear transmutation when exposed to a neutron flux, were placed exterior of the chamber. Following exposures of the order of days or weeks, the experimentalists reported nuclear transmutations that were based on the observed neutron counts of up to $104 \mathrm{cps}$. Don Borghi experiment has been strongly criticized by academia on pure theoretical grounds without the actual repetition of the tests. Note that experiment makes no claim of direct detection of neutrons, and only claims the detection of clear nuclear transmutations.

To verify the claim of Don Borghi's experiment, Santilli repeated this experiment in large number of laboratories and institutions the world over.

\section{Santilli Experiment on the Synthesis of Neutrons}

Santilli conceived his experiment [15], [16] as being solely based on the use of an electric arc within a cold (i.e., at atmospheric temperature) hydrogen gas without any use of microwave at all. Three different klystrons were manufactured, tested and used for the measurements. The specifications of detectors were used for measurements are given below:

1. A detector model PM1703GN manufactured by Polimaster, Inc., with sonic and vibration alarms as well as memory for printouts, with the photon channel activated by $\mathrm{CsI}$ and the neutron channel activated by LiI.

2. A photon-neutron detector SAM 935 manufactured by Berkeley Nucleonics, Inc., with the photon channel activated by $\mathrm{NaI}$ and the neutron channel activated by He-3 also equipped with sonic alarm and memory for printouts of all counts. This detector was used to verify the counts from the preceding one.

3. A BF3 activated neutron detector model 12-4 manufactured by Ludlum Measurements, Inc., without counts memory for printouts. This detector was used to verify the counts by the preceding two detectors.

Electric arcs were powered by welders manufactured by Miller Electric, Inc., including a Syncrowave 300, a Dynasty 200 , and a Dynasty 700 capable of delivering an arc in DC or $\mathrm{AC}$ mode, the latter having frequencies variable from 20 to $400 \mathrm{~Hz}$.

Klystron-I was cylindrical and sealed, of about 6" outside diameter and 12" height, made of commercially available, transparent, PolyVinyl Chloride (PVC) housing along its symmetry axis a pair of tungsten electrodes. The electrodes gap was controllable by sliding the top conducting rod through the seal of the flange. The klystron cylindrical wall was transparent so as to allow a visual detection of arc. After initiation of DC arc there was no detection for hours. However, shaking of klystron the neutrons were detected in a systematic and repetitive way. The detection was triggered by a neutron-type particle, excluding contributions from photons. However, these detections were anomalous, that is, they did not appear to be due to a flux of actual neutrons originating from the klystron. This anomaly is established by the repeated "delayed detections," that is, exposure of the detector to the 
klystron with no counts of any type, moving the detector away from the klystron (at times for miles), then seeing the detectors enter into off-scale vibrations and sonic alarms with zero photon counts.

Klystron-II was a rectangular, transparent, made up of PVC of dimension. This klystron was small in size than earlier one to avoid implosion caused by combustion with atmospheric oxygen. This test was conducted only once because of instantaneous off-scale detection of neutrons by all detectors which led to evacuation of the laboratory. Hence, this test was not repeated for safety.

Klystron-III was cylindrical made up of carbon steel pipe with 12 " outer diameter, 0.5 " wall thickness, 24 " length and 3" thick end flanges to sustain hydrogen pressure up to 500 psi with the internal arc between throated tungsten electrodes controlled by outside mechanisms. This test was conceived for the conduction of the test at bigger hydrogen pressure compared to that of Klystron I. The test was conducted only once at 300 psi hydrogen pressures because of instantaneous, off-scale, neutron detections such to cause another evacuation of the laboratory.

The main purpose of Santilli's of conducting these tests was to establish the production of neutron-type particles via a DC arc within a hydrogen gas. He has experimented identical tests with other gases, but no meaningful counts were detected other than hydrogen. No neutron, photon or other radiation was measured from electric arcs submerged within liquids. Hence, the reported findings appear to be specific for electric arcs within a hydrogen gas under the conditions stated above.

\section{The Don Borghi-Santilli Neutroid}

Santilli $[5,15]$ excludes that the entities produced in the tests with Klystron I are true neutrons for various reasons, such as:

1. The anomalous behavior of the detector, in the case of the 15 minute delay, namely the self-activated detection indicates first the absorption of "entities" producing nuclear transmutations that, in turn release ordinary neutrons.

2. The environment inside stars can indeed provide the missing energy of $0.78 \mathrm{MeV}$ for the neutron synthesis, but the environment inside Klystron-I cannot do the same due to the very low density of the hydrogen gas.

3. The physical laws of hadronic mechanics do not allow the synthesis of the neutron under the conditions of Klystron-I because of the need of the trigger, namely, an external event permitting the transition from quantum to hadronic conditions. In fact, the tests with Klystrons-II and III do admit the trigger required by hadronic mechanics. However, Santilli did not discard that the "entities" produced in the tests with Klystrons-II and III are indeed actual neutrons, due to the instantaneous, off-scale nature of the neutron alarms in clear absence of photon or vibrations.

In view of above reasons, Don Borghi [13], [14] submitted the hypothesis that the "entities" are neutron-type particles called "neutroids". Santilli adopted this hypothesis and presented the first technical characterization of neutroids with the symbol, $\tilde{n}$ and the characteristics in conventional nuclear units, $A=1, Z=0, J=0, a m u=0.008$. Hence, Santilli assumed that in Klystron-I, he produced the following reaction precisely along Rutherford's original conception

$$
p^{+}+e^{-} \rightarrow \tilde{n}(1.0,0,1.008)
$$

where the value $J=0$ is used for the primary purpose of avoiding the spin anomaly in the neutron synthesis as indicated above and the rest energy of the neutroids is assumed as being that of the hydrogen atom.

\section{Interpretation of Don Borghi and Santilli Experiments}

In Don Borghi's and Santilli's experiments the various substances placed in the exterior of the klystrons did indeed experience nuclear transmutations. If we discard the Don Borghi's klystron and Santilli's Klystron-I to produce actual neutrons, then the main question arises from where the neutrons originated and detected. Evidently, only two possibilities remain, namely, that the detected neutrons were actually synthesized in the walls of the klystrons, or by the activated substances themselves following the absorption of the neutroids produced by the klystrons. Considering the neutrino hypothesis has no sense for the neutron synthesis for various reasons, Santilli $[5,15]$ assumes that the energy, spin and magnetic anomalies in the neutron synthesis are accounted for by their transfer either from nuclei or from the aether via his etherino hypothesis

$$
\tilde{n}(1.0,0,1.008)+a \rightarrow n(1.0,0,1.008) .
$$

Assuming the binding energy of a neutroid is similar to that of an ordinary nucleon (since neutroids are assumed to be converted into neutrons when inside nuclei, or to decompose into protons and electrons, thus recovering again the nucleon binding energy), Santilli indicates the following possible nuclear reaction for one of the activated substances in Don Borghi's tests

$$
\begin{array}{r}
A u(197,79,3 / 2,196.966)+\tilde{n}+a \\
\rightarrow A u(198,79,2,197.972)
\end{array}
$$

produces known nuclide, hence it indicates that neutrons were synthesized by the activating substances themselves on absorption of neutroid. The nuclear reaction with steel wall of the klystron,

$$
\begin{array}{r}
F e(57,26,1,57.935)+\tilde{n}+a \\
\rightarrow F e(58,26,1,57.941)
\end{array}
$$

yields an unknown nuclide, $F e(58,26,1,57.941)$ because the known nuclide is $F e(58,26,0,57.933)$. This indicates that the neutrons in Don Borghi experiment were not 
synthesized in the walls of his klystron. Eq.(2) also allow an interpretation of some of Santilli detections [5], [15], with the understanding that the anomalous behavior of the detectors, such as the delayed neutron counts, requires special studies and perhaps the existence of some additional event not clearly manifested in Don Borghi's tests.

To initiate the study, Santilli considered the first possible reaction inside the klystron

$$
\begin{array}{r}
H(1,1,1 / 2,1.008)+\tilde{n}+a \\
\rightarrow H(1,1,1,2.014),
\end{array}
$$

delivers ordinary deuteron on coupling of hydrogen atom and neutroid. This indicates neutrons cannot be originated inside the klystron-I. Next, Santilli considered following nuclear reactions with the polycarbonate of Klystron-I wall containing about 75 percent carbon and 18.9 percent oxygen

$$
\begin{aligned}
& C(12,6,0,12.00)+\tilde{n}+a \\
& \rightarrow C(13,6,1 / 2,13.006) \\
& \rightarrow C(13,6,1 / 2,13.006)+\gamma, \\
& O(16,8,0,16.00)+\tilde{n}+a \\
& \rightarrow O(17,8,1 / 2,17.006),
\end{aligned}
$$

do not give conventional activation processes. Thus, in Santilli's experiment too, it does not appear that the detected neutrons are synthesized by the walls of klystron. The above analysis leads us to the only remaining possibility that in Santilli tests, the neutrons are synthesized by the detectors themselves. To study this possibility, Santilli considered the reaction using Li-activated detectors,

$$
\begin{aligned}
& \operatorname{Li}(7,3,3 / 2,7.016)+\tilde{n}+a \\
& \rightarrow \operatorname{Li}(8,3,2,8.022) \\
& \rightarrow \operatorname{Be}(8,4,0,8.005)+e^{-} \rightarrow 2 \alpha,
\end{aligned}
$$

that behaves fully equivalent to detection of neutriods or neutrons. This indicated that neutrons detected in Santilli experiment were synthesized by the substance used for detection after absorption of neutriods.

\section{Concluding Remarks}

It is observed that Santilli's discovery of hadronic mechanics appropriately represents, at both non-relativistic and relativistic levels, "all" characteristics of neutron according to Rutherford's conjecture of its synthesis from hydrogen atom in the core of a star. A first implication of the studies is that the orbital motion of the electron within the hyperdense proton allows the conservation of the total angular momentum without any need for the conjecture of the hypothetical neutrino. Another important implication is the dismissal of quarks as the actual physical constituents of the neutron since the proton and the electron cannot "disappear" at the time of the neutron synthesis to be replaced by the hypothetical quarks, and then "reappear" at the time of the neutron decay. We show that, besides the above mathematical and theoretical studies, Santilli has provided numerous experimental verification of the laboratory synthesis of the neutron from a hydrogen gas in support of Rutherford's historical hypothesis.

\section{Acknowledgments}

The author is highly grateful to Professor R. M. Santilli, his team and Professor Anderson for valuable guidance in preparing and for suggestions to improve this paper. The financial support from the R. M. Santilli Foundation is gratefully acknowledged. The author is also highly indebted to Professor A. A. Bhalekar for suggesting me this topic and helping me in writing this paper by his valuable advices and suggestions in its overall improvement.

\section{References}

[1] H. Rutherford, Proc. Roy. Soc. A, 97, 374 (1920).

[2] J. Chadwick, Proc. Roy. Soc. A, 136, 692 (1932).

[3] W. Pauli, Handbuch der Physik, 24, Berlin, Springer Verlag, 1933.

[4] E. Fermi, Nuclear Physics, Chicago, University of Chicago Press, 1949.

[5] R. M. Santilli, Hadronic Mathematics, Mechanics and Chemistry, Vol. I-V, International Academic Press, Palm Harbor, U.S.A., 2008.

[6] R. M. Santilli, "Apparent Consistency of Rutherford's Hypothesis on Neutron as a Compressed Hydrogen Atom", Hadronic J. 13, 513-531(1900).

[7] R. M. Santilli, "Apparent Consistency of Rutherford's Hypothesis on Neutron Structure via Hadronic Generalization of Quantum Mechanics-I: Nonrelativistic Treatment", in ICTP-1991, International Academic Press, Palm Harbor, Florida, 1992.

[8] I. Gandzha and J. Kadeisvily, New Science for A New Era, Sankata Printing Press, Kathmandu, Nepal, 2001.http://www.santilli-foundation.org/docs/ RMS

[9] R. M. Santilli,Lie-admissible Approach to the Hadronic Structure, Volume I: Non-applicability of the Galilei and Einstein Relativities in the series Monographs in Theoretical Physics, Hadronic Press, Palm Harbor, Florida, 1978.

[10] R. M. Santilli, Lie-admissible Approach to the Hadronic Structure, Volume II: Coverings of the Galilei and Einstein Relativities in the series Monographs in Theoretical Physics, Hadronic Press, Palm Harbor, Florida, 1981.

[11] R. M. Santilli, ÓNonlocal-Integral Isotopies of Differential Calculus, Mechanics and Geometries,Ó in Isotopies of Contemporary Mathematical Structures, Rendiconti Circolo Matematico Palermo, Suppl. Vol. 42, 7-82 (1996), http://www.santilli-foundation.org/docs/Santilli-37.pdf

[12] R. M. Santilli, "The etherino and/or the neutrino hypothesis", Foundation of Physics, 37, 670 (2007).

[13] C. Borghi, C. Giori C. and A. Dall'Olio, Communications of CENUFPE, 8(1969) and 25 (1971). 
[14] C. Borghi, C. Giori C. and A. Dall'Olio, (in Russian), Phys. Atomic Nuclei, 56, 205 (1993).

[15] R. M. Santilli, "Apparent Confirmation of Don Borghi's Experiment toward the Synthesis of Neutron from Protons and Electrons", Hadronic Journal, textbf30, 709 (1997).
[16] R. M. Santilli, The Synthesis of the Neutron, http://www.neutronstructure.org /neutronsynthesis.htm. 